\title{
Bicanonical map of surfaces with $\chi=1$ fibered by hyperelliptic curves of genus 3
}

\author{
Giuseppe Borrelli* \\ (Communicated by R. Miranda)
}

\begin{abstract}
We investigate surfaces of general type $S$ with $\chi(S)=1$ that admit a fibration $f: S \rightarrow$ $\mathbb{P}^{1}$ whose general fiber is a hyperelliptic curve of genus 3 . We show that the bicanonical map of $S$ factors through a generically finite rational map of degree two onto a ruled surface.

Together with a previous result, this yields a characterization of surfaces with $\chi=1$ whose bicanonical map factors through a $2: 1$ map onto a ruled surface in terms of the existence of fibrations of small genus.
\end{abstract}

\section{Introduction}

The classification problem for algebraic surfaces leads naturally to the study of fibrations $f: S \rightarrow \mathbb{B}$ from a surface onto a smooth curve whose general fiber is a smooth and connected curve of prescribed genus $g$. For instance, fibrations with $g \leq 1$ are essential in the Enriques classification.

Let $S$ be a surface of general type, and $f: S \rightarrow \mathbb{B}$ a fibration over a curve. Then a general fiber of $f$ has genus $g \geq 2$. The limit case $g=2$ plays a special role in the classification of surfaces whose bicanonical map $\varphi_{2 K_{S}}$, i.e., the rational map associated to the linear system $\left|2 K_{S}\right|$, is not birational. Indeed, since the restriction of $\varphi_{2 K_{S}}$ to a general fiber of $f$ factors through the bicanonical map of the fiber itself, if $g=2$, then $\varphi_{2 K_{S}}$ cannot be birational. Surfaces admitting such a fibration are said to present the standard case for the nonbirationality of $\varphi_{2 K_{S}}$.

The study of the behavior of the bicanonical map is a useful tool in the classification of surfaces of general type. By a result of Bombieri in [6] refined by Reider in [25] we know that, if $S$ is minimal, $K_{S}^{2} \geq 10$ and the bicanonical map fails to be birational, then $S$ presents the standard case. Surfaces with nonbirational bicanonical map not presenting the standard case have been intensively investigated in the last decade by several authors, and their classification is rather understood with the exception of the cases when $p_{g}=$

${ }^{*}$ This work was partially supported by bolsa DTI-Institutodo Milenio/CNPq. 
$q \leq 1$, cf. [11] and [2] for a survey on this subject, see also [22] and [26] for recent work on surfaces with $p_{g}=q=1$.

Let $S$ be a surface of general type with $p_{g}=q \leq 1$. Suppose that the bicanonical map factors through a 2:1 map onto a ruled surface and $S$ does not present the standard case. Then, by [7, Theorem 0.4], there exists a fibration $f: S \rightarrow \mathbb{P}^{1}$ whose general fiber is a hyperelliptic curve of genus 3 . In the present paper we show the converse, that is, if $S$ admits a fibration $f: S \rightarrow \mathbb{P}^{1}$ with general fiber a hyperelliptic curve of genus 3, then $\varphi_{2 K_{S}}$ factors through a double cover of a ruled surface. More precisely, we prove the following.

Theorem 0.1. Let $S$ be a surface of general type, and $f: S \rightarrow \mathbb{P}^{1}$ a fibration. If $\chi(S)=$ 1 and the general fiber of $f$ is a hyperelliptic curve of genus 3 , then one of the following cases occurs:

(1) the bicanonical map of $S$ factors through the involution induced by the hyperelliptic involution of the fibers of $f$;

(2) $p_{g}=q=1, K_{S}^{2}=2$ and $S$ is minimal.

In particular, the bicanonical map of $S$ factors through a generically finite rational map $S \rightarrow W$ of degree two onto a ruled surface $W$. In the first case $q(W)=0$, while in the second case $q(W)=1$.

An application of Theorem 0.1 is the following characterization of minimal surfaces with $p_{g}=q \leq 1$ whose bicanonical map factors through a generically finite map of degree two onto a ruled surface in terms of the existence of fibrations of small genus.

Theorem 0.2. Let $\tilde{S}$ be a minimal surface of general type. Suppose that $p_{g}=q \leq 1$.

Then the following are equivalent:

(1) the bicanonical map of $\tilde{S}$ factors through a generically finite rational map of degree two onto a ruled surface;

(2) there exists a birational morphism $S \rightarrow \tilde{S}$ and a fibration $f: S \rightarrow \mathbb{B}$ over a smooth curve $\mathbb{B}$ of genus $b \leq q$ such that a general fiber of $f$ is a smooth hyperelliptic curve of genus $g \leq 3-b$.

In particular, by combining this with previous results in [14], [15], [16], [19] and [17], it follows that the bicanonical map of a minimal surface of general type $\tilde{S}$ with $p_{g}=q=0$ and $K_{\tilde{S}}^{2} \geq 6$ fails to be birational if and only if there is a fibration $f: \tilde{S} \rightarrow \mathbb{P}^{1}$ whose general fiber is a hyperelliptic curve of genus 3 (see Corollary 3.1).

The paper is organized as follows. In Section 1 we gather some facts concerning involutions, and we fix some notation. In Section 2, which is the core of the paper, we prove some technical lemmas, and then we break the proof of Theorem 0.1 into three parts. In Section 3, after finishing the proof of Theorem 0.1, we prove Theorem 0.2 and a corollary. In the last section we give a list of some known examples of the above situation.

I would like to thank the referee for his/her useful suggestions and remarks. 
0.1 Notation. We work over the field of complex numbers. Given a smooth surface $X$, we denote by $K_{X}$ a canonical divisor of $X$ and use the standard notation $p_{g}(X)=$ $h^{0}\left(K_{X}\right), q(X)=h^{1}\left(K_{X}\right)$ and $\chi(X)=p_{g}(X)-q(X)+1$. We use the symbols $\sim$ and $\equiv$ to denote respectively numerical and linear equivalence of divisors. As usual, a $(-n)$ curve on a surface is a smooth rational curve with self intersection $-n$. An involution on a variety is an automorphism of order 2 .

$S$ will always denote a surface of general type and $\varphi: S \rightarrow \tilde{S}$ the birational morphism to its minimal model. For the sake of brevity, we write $p_{g}$ for $p_{g}(S)$ and $q$ for $q(S)$. Note that $p_{g}=p_{g}(\tilde{S})$ and $q=q(\tilde{S})$.

A fibration is a surjective morphism with connected fibers from a smooth surface onto a smooth connected curve. By a (hyperelliptic) fibration of genus $g$ we mean a fibration whose general fiber is a (hyperelliptic) curve of genus $g$. A fibration is said to be relatively minimal if the fibers contain no $(-1)$-curves, and it is said to be smooth if every fiber is smooth.

Given a variety $X$ and two rational maps $h_{1}: X \rightarrow V_{1}, h_{2}: X \rightarrow V_{2}$, we say that $h_{2}$ factors through $h_{1}$ if either $V_{1}=X$ and $h_{2}=h_{2} \circ h_{1}$, or $V_{1} \neq X$ and there exists a rational map $h_{3}: V_{1} \rightarrow V_{2}$ such that $h_{2}=h_{3} \circ h_{1}$.

For any rational number $a$, we denote by $\lfloor a\rfloor$ the round-down of $a$, i.e. the greatest integer less than or equal to $a$.

\section{Generalities on involutions and setup}

We will fix notation and gather some facts concerning involutions. Throughout the present section we make the following assumption.

Assumption 1.1. Let $S$ be a smooth surface of general type with $\chi(S)=1$, and let $f: S \rightarrow \mathbb{P}^{1}$ be a relatively minimal hyperelliptic fibration of genus 3 .

1.1. Let $F$ be a general fiber of $f$, and denote by $\sigma_{F}$ the hyperelliptic involution on $F$. Since $f: S \rightarrow \mathbb{P}^{1}$ is relatively minimal, there exists an involution $\sigma$ such that $\left.\sigma\right|_{F}=\sigma_{F}$ for any general fiber $F$.

Let $\varphi: S \rightarrow \tilde{S}$ be the birational morphism to the minimal model. Since $\tilde{S}$ is minimal, $\tilde{\sigma}=\varphi \circ \sigma \circ \varphi^{-1}$ is an involution on $\tilde{S}$. Let $\tilde{F}=\varphi_{*} F$. Then, since $f$ is relatively minimal, $F$ is the strict transform of $\tilde{F}$. In particular if $\tilde{F}$ is smooth then $K_{\tilde{S}}^{2}-K_{S}^{2}=\tilde{F}^{2}$.

1.2. The fixed locus of $\sigma$ (respectively $\tilde{\sigma}$ ) is the union of a, possibly reducible, curve $R$ (respectively $\tilde{R}$ ) and a possibly empty finite set $\left\{Q_{1}, \ldots, Q_{\nu}\right\}$ (respectively $\left\{P_{1}, \ldots, P_{\tilde{\nu}}\right\}$ ) of isolated points. By the Hurwitz formula for the double cover $F \rightarrow F / \sigma_{F} \cong \mathbb{P}^{1}$, we have $R \cdot F=8$. Notice that, by construction, $\tilde{R}=\varphi_{*} R$.

1.3. The quotient $Y=S / \sigma$ (respectively $\tilde{Y}=\tilde{S} / \tilde{\sigma}$ ) is a surface that is singular at the images of the $Q_{i}$ 's (respectively $P_{i}$ 's), which are pairwise distinct nodes (i.e., rational double points of type $A_{1}$ ), and is smooth elsewhere. 
Let $\tilde{\pi}: \hat{S} \rightarrow \tilde{S}$ be the blow up of $P_{1}, \ldots, P_{\tilde{\nu}}$, and $E_{1}, \ldots, E_{\tilde{\nu}}$ the exceptional curves of $\tilde{\pi}$ lying over the points $P_{1}, \ldots, P_{\tilde{\nu}}$ respectively. Let $\hat{\Sigma} \rightarrow \tilde{Y}$ be the minimal desingularization of $\tilde{Y}$. Then the $E_{i}$ 's are $(-1)$-curves, and $\hat{\sigma}=\tilde{\pi} \circ \sigma \circ \tilde{\pi}^{-1}$ is an involution on $\hat{S}$ whose fixed locus is the smooth curve $\hat{R}=\tilde{\pi}^{*} \tilde{R}+\sum E_{i}$. The quotient $\hat{S} / \hat{\sigma}$ is isomorphic to $\hat{\Sigma}$, and we have a commutative diagram

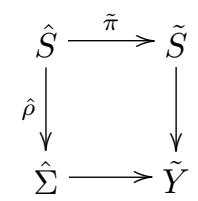

where $\hat{\rho}: \hat{S} \rightarrow \hat{\Sigma} \cong \hat{S} / \hat{\sigma}$ is the projection to the quotient. Hence $\hat{\rho}$ is a double cover branched along the smooth curve $\hat{B}=\hat{\rho}(\hat{R})$, and $\hat{B} \in|2 \hat{\Delta}|$ for some $\hat{\Delta} \in \operatorname{Pic}(\hat{\Sigma})$.

Let $\hat{F}$ be the strict transform of $\tilde{F}$ on $\hat{S}$. Then the image of $\hat{F}$ on $\hat{\Sigma}$ is a curve birational to $F / \sigma_{F} \cong \mathbb{P}^{1}$, and so $\hat{\Sigma}$ is a rational surface.

1.4. Let $X$ be a surface of general type, $\varphi_{2 K_{X}}: X \rightarrow X_{[2]}$ its bicanonical map, and $\tau$ an involution on $X$. We say that the bicanonical map $\varphi_{2 K_{X}}$ of $X$ factors through $\tau$ if $\varphi_{2 K_{X}}=\varphi_{2 K_{X}} \circ \tau$. We will use the following

Proposition 1.2 (cf. [7, Proposition 1.8]). Let $\tilde{S}, \tilde{\sigma}$ and $\hat{\Sigma}$ be as in 1.1-1.3. Then

$$
\begin{aligned}
& \tilde{\nu}=K_{\tilde{S}}^{2}-2 \chi(\tilde{S})+6 \chi(\hat{\Sigma})-2 h^{0}\left(2 K_{\hat{\Sigma}}+\hat{\Delta}\right), \\
& \tilde{\nu}=\tilde{R} \cdot K_{\tilde{S}}-4 \chi(\tilde{S})+8 \chi(\hat{\Sigma}),
\end{aligned}
$$

and the bicanonical map of $\tilde{S}$ factors through $\tilde{\sigma}$ if and only if $h^{0}\left(2 K_{\hat{\Sigma}}+\hat{\Delta}\right)=0$.

1.5 Canonical resolution. Let the notation be as above. Given a birational morphism $\psi: \hat{\Sigma} \rightarrow \Sigma$, let $B:=\psi_{*} \hat{B}$ and $\Delta=\psi_{*} \hat{\Delta}$. Notice that $B \in|2 \Delta|$. Set $d:=K_{\Sigma}^{2}-K_{\hat{\Sigma}}^{2}$.

Suppose that $\psi$ is not an isomorphism. Then, by [7, Proposition 1.6], $B$ is a singular curve and $\hat{S}$ is the canonical resolution of the double cover of $\Sigma$ branched along $B$. Set $\Sigma_{0}=\Sigma$ and for each $i \in\{1, \ldots, d\}$ let $\psi_{i}: \Sigma_{i} \rightarrow \Sigma_{i-1}$ be the blow up at a point $q_{i-1}$ so that $\psi=\psi_{1} \circ \cdots \circ \psi_{d}$. Let $\mathcal{E}_{i}$ be the exceptional curve of $\psi_{i}$ and denote by $\mathcal{E}_{i}^{*}$ its pull back to $\hat{\Sigma}$. Set $B_{0}=B$ and, having defined $B_{i-1} \subset \Sigma_{i-1}$, define

$$
B_{i}=\psi_{i}^{*} B_{i-1}-2\left\lfloor\frac{m_{i-1}}{2}\right\rfloor \mathcal{E}_{i}, \quad \text { for } i \geq 1,
$$

where $m_{i-1}$ is the multiplicity at $q_{i-1}$ of $B_{i-1}$. Then $m_{i} \geq 2$ for every $i \geq 0$ and we have

$$
\hat{B}=\psi^{*} B-\sum_{i=1}^{d} 2\left\lfloor\frac{m_{i-1}}{2}\right\rfloor \mathcal{E}_{i}^{*} .
$$


Let $\bar{B}_{i-1}$ be the strict transform of $B_{i-1}$ on $\Sigma_{i}$. Then observe that $B_{i}=\bar{B}_{i-1}$ if $m_{i-1} \equiv 0$ $(\bmod 2)$ and $B_{i}=\bar{B}_{i-1}+\mathcal{E}_{i}$ otherwise. We have

$$
\begin{aligned}
\chi(\hat{S}) & =\frac{1}{2}\left(K_{\Sigma}+\Delta\right) \cdot \Delta+2 \chi(\Sigma)-\frac{1}{2} \sum\left\lfloor\frac{m_{i}}{2}\right\rfloor\left(\left\lfloor\frac{m_{i}}{2}\right\rfloor-1\right), \text { and } \\
K_{\hat{S}}^{2} & =2\left(K_{\Sigma}+\Delta\right)^{2}-2 \sum\left(\left\lfloor\frac{m_{i}}{2}\right\rfloor-1\right)^{2} .
\end{aligned}
$$

Note that the multiplicity of $B_{i}$ at any point $y \in \mathcal{E}_{i}$ is less than or equal to $m_{i-1}+1$, and that equality holds if and only if $m_{i-1} \equiv 1(\bmod 2)$ and $\bar{B}_{i-1}$ has multiplicity $m_{i-1}$ at $y$.

Definition 1.3. If $m_{i-1}=k$ is odd (and hence $\geq 3$ ) and $B_{i}=\bar{B}_{i-1}+\mathcal{E}_{i}$ has multiplicity $m_{i-1}+1$ at a point $y$ lying on $\mathcal{E}_{i}$, then we say that $B$ has a $[k, k]$-point at $q_{i-1}$. Since $B$ is smooth, without loss of generality we may, and we shall, choose the $\psi_{i}$ 's so that if $B$ has a $[k, k]$-point at $q_{i-1}$, then $q_{i}$ is the point lying on $\mathcal{E}_{i}$ where $B_{i}=\bar{B}_{i-1}+\mathcal{E}_{i}$ has multiplicity $k+1$.

If $B$ does not have a $[k, k]$-point at $q_{i}$ nor at $q_{i-1}$, we say that $B$ has a $\left[k^{\prime}\right]$-point at $q_{i}$, where $k^{\prime}=m_{i}$ if $m_{i} \equiv 0(\bmod 2)$ and $k^{\prime}=m_{i}-1$ otherwise.

Set $\bar{a}=\left(a_{2}, a_{3}, a_{4}, \ldots\right)$, where $a_{k}$ is the number of $[k]$-points if $k \equiv 0(\bmod 2)$ and the number of $[k, k]$-points if $k \equiv 1(\bmod 2)$.

Since $B$ is smooth and $m_{i} \geq 2$, it is straightforward to see that

$$
d=\sum_{r \geq 1}\left(2 a_{2 r+1}+a_{2 r}\right)
$$

1.6. Suppose that $\varphi: S \rightarrow \tilde{S}$ is an isomorphism. Then $\tilde{F}=F$ and $\hat{F}=\pi^{*} F$. The composite morphism $\hat{f}=f \circ \pi: \hat{S} \rightarrow \mathbb{P}^{1}$ yields a fibration whose general fiber is $\hat{F}$. Let $\hat{\Gamma}=\hat{\rho}(\hat{F}) \subset \hat{\Sigma}$. Then $\hat{\Gamma}$ is a smooth rational curve and $\hat{h}=\hat{f} \circ \hat{\rho}^{-1}: \hat{\Sigma} \rightarrow \mathbb{P}^{1}$ is a fibration whose general fiber is $\hat{\Gamma}$, i.e., $\hat{\Sigma}$ is a rational ruled surface.

By [29, Lemma 6] there exists a birational morphism $\psi: \hat{\Sigma} \rightarrow \Sigma$ such that $\Sigma$ is geometrically ruled and the following holds.

(i) $\Gamma=\psi_{*} \hat{\Gamma}$ is a ruling of $\Sigma$ and $\Gamma \cdot B=8$, where $B=\psi_{*} \hat{B}$.

(ii) $a_{k}=0$ if $k \geq 6$ (cf. 1.5 and Definition 1.3), i.e., $B$ has at most [5,5]-points and $\bar{a}=\left(a_{2}, a_{3}, a_{4}, a_{5}\right)$.

(iii) If $B$ has a [5,5]-point at $q_{i}$ then $\psi_{1} \circ \cdots \circ \psi_{i}: \Sigma_{i} \rightarrow \Sigma$ is an isomorphism in a neighborhood of $q_{i}$, and the ruling of $\Sigma$ through the image of $q_{i}$ is a component of $B$.

Lemma 1.4 (cf. [29, Lemma 7 and Formula (3), p. 606]). Let the notation be as above and suppose that $\varphi: S \rightarrow \tilde{S}$ is an isomorphism.

Let $\psi: \hat{\Sigma} \rightarrow \Sigma$ be a birational morphism to a geometrically ruled surface satisfying (i), (ii) and (iii) above. Then

$$
\tilde{\nu}=K_{\tilde{S}}^{2}-K_{\hat{S}}^{2}=2 a_{5}+a_{3} .
$$




\section{Hyperelliptic fibrations of genus 3 and bicanonical map}

Throughout the present section we assume $S$ and $f: S \rightarrow \mathbb{P}^{1}$ as in Assumption 1.1, and use the setup and notation introduced in 1.1, 1.2, 1.3.

We start by providing some information on $\tilde{S}$ and the self-intersection of $\tilde{F}=\varphi_{*} F$.

Lemma 2.1. Under the current assumptions, one of the following occurs:

(1) $\tilde{F}^{2}=4, \tilde{F}$ has a double point and $2 K_{\tilde{S}} \sim \tilde{F}$. In this case $q=0$.

(2) $\tilde{F}^{2}=2, \tilde{F}$ is smooth and $K_{\tilde{S}}^{2}=1$. In this case $q=0$.

(3) $\tilde{F}^{2}=2, F$ is smooth and $K_{\tilde{S}} \sim \tilde{F}$. In this case $q=1$.

(4) $\tilde{F}^{2} \leq 1$ and $\tilde{F}$ is smooth. In this case $q \leq 2$.

Proof. Since the general fiber of $f: S \rightarrow \mathbb{P}^{1}$ has genus 3, by [5, Lemme] we have $q \leq 3$, and equality holds if and only if $S$ is birationally equivalent to $F \times \mathbb{P}^{1}$. Thus $q \leq 2$.

If $\tilde{F}^{2} \leq 1$, then $\tilde{F}$ is clearly smooth. Assume $\tilde{F}^{2} \geq 2$. Then $\varphi: S \rightarrow \tilde{S}$ is the blow up at $n \geq 1$ points $x_{1}, \ldots, x_{n}$, and $F$ is the strict transform of $\tilde{F}$. Let $\mu_{i}$ be the multiplicity of $\tilde{F}$ at $x_{i}$. Then $\mu_{i} \geq 1$, and we have

$$
4=F \cdot K_{S}=\tilde{F} \cdot K_{\tilde{S}}+\sum \mu_{i}>\sum \mu_{i},
$$

because $\tilde{S}$ is minimal of general type and $h^{0}(\tilde{F}) \geq 2$. Note that $\sum \mu_{i} \geq 2$. Therefore, by the algebraic index theorem, we get

$$
4 \geq\left(4-\sum \mu_{i}\right)^{2}=\left(\tilde{F} \cdot K_{\tilde{S}}\right)^{2} \geq \tilde{F}^{2} \cdot K_{\tilde{S}}^{2} \geq \tilde{F}^{2}
$$

Suppose that $\tilde{F}$ is singular. Then we have $\tilde{F}^{2} \geq 4$. Hence (2.2) yields $\tilde{F}^{2}=4$, $K_{\tilde{S}}^{2}=1$ and $\tilde{F} \sim 2 K_{\tilde{S}}$. By [12, Théorème 6.1], any minimal surface of general type $X$ with $q(X)>0$ satisfies $K_{X}^{2} \geq 2 q(X)$. Thus $q=0$ and we have Case (1).

Now suppose that $\tilde{F}$ is smooth. Then, by (2.2) and the adjunction formula, we get $\tilde{F}^{2}=2$ and $K_{\tilde{S}}^{2} \leq 2$, and equality holds if and only if $\tilde{F} \sim K_{\tilde{S}}$. As before, $K_{\tilde{S}}^{2}=1$ yields $q=0$ and we have Case (2).

Suppose $K_{\tilde{S}}^{2}=2$. If $q \geq 1$, then $2=K_{\tilde{S}}^{2} \geq 2 q$ (loc.cit.) yields $q=1$. Assume $q=0$. Then $\eta=\tilde{F}-K_{\tilde{S}}$ defines a nonzero torsion element in $\operatorname{Pic}(\tilde{S})$. An obvious calculation yields $h^{1}\left(K_{\tilde{S}}+\eta\right)>0$, implying that the étale cover $Y \rightarrow \tilde{S}$ associated to $\eta$ has $q(Y)>0$. This contradicts [3, Corollaire 5.8]. Therefore $q=1$ and we have Case (3).

We will proceed by considering three distinct situations: the first one is $q>1$, the second one is $\tilde{F}^{2}>0$, and the final is that in which $q \leq 1$ and $\tilde{F}^{2}=0$.

2.1. In this section we deal with the cases $q>1$ and $\tilde{F}^{2}>0$.

Proposition 2.2. Suppose $q>1$. Then

(1) $p_{g}=q=2$ and $K_{S}^{2}=K_{\tilde{S}}^{2}=8$, 
(2) the Albanese morphism of $\tilde{S}$ is a smooth and isotrivial fibration of genus 2,

(3) the bicanonical map of $\tilde{S}$ factors through $\tilde{\sigma}$.

Proof. By Lemma 2.1 we have $q=2$ and $K_{\tilde{S}}^{2}-K_{S}^{2}=\tilde{F}^{2} \in\{0,1\}$. Let $A=\operatorname{Alb}(\tilde{S})$ be the Albanese variety of $S$. Then $A$ is an abelian surface and so, by [21, Theorem 1 and Remark 1], any linear system on $A$ contains only a finite number of elliptic or hyperelliptic curves. It follows that the Albanese morphism $\alpha: \tilde{S} \rightarrow A$ of $\tilde{S}$ is not surjective, and so it yields a fibration over a smooth curve of genus $2=q(\tilde{S})$.

Let $m$ be the genus of a general fiber of $\alpha$. Since $\tilde{S}$ is of general type, $m \geq 2$ and, by [5, Corollaire], we have $8(m-1) \leq K_{\tilde{S}}^{2}$. Moreover, by Miyaoka-Yau's inequality, $K_{\tilde{S}}^{2} \leq 9 \chi(\tilde{S})$. Thus $m=2$ and $8 \leq K_{\tilde{S}}^{2} \leq 9$. By [28, Corollaire, p. 18] a surface $X$ fibered by curves of genus 2 satisfies $K_{X}^{2} \leq 8 \chi(X)$, and equality holds if and only if the fibration is smooth and isotrivial. Hence $K_{\tilde{S}}^{2}=8$, and the Albanese morphism of $\tilde{S}$ is a smooth and isotrivial fibration of genus 2 .

Let $C$ be a fiber of $\alpha: \tilde{S} \rightarrow \alpha(\tilde{S})$. Suppose that $\tilde{F}^{2}=1$. Then $K_{\tilde{S}} \cdot(\tilde{F}+C)=5$ and thus, by the algebraic index theorem and $K_{\tilde{S}}^{2}=8$, we obtain $(\tilde{F}+C)^{2} \leq 3$, an obvious contradiction.

Thus $\tilde{F}^{2}=0$. Notice that in this case, with the same argument, we obtain $K_{\tilde{S}} \sim$ $\tilde{F}+2 C$. Hence $\tilde{F} \cdot C=2$ and $\tilde{F}^{2}=0$. In particular, $\varphi: S \rightarrow \tilde{S}$ is an isomorphism and $K_{S}^{2}=K_{\tilde{S}}^{2}=8$.

In order to prove that the bicanonical map of $\tilde{S}$ factors through the involution $\tilde{\sigma}$, it suffices to show that $h^{0}\left(2 K_{\hat{\Sigma}}+\hat{\Delta}\right)=0$. Since $\hat{\Sigma}$ is rational, by (1.2) and (1.3) we have

$$
\tilde{F} \cdot \tilde{R} \leq(\tilde{F}+2 C) \cdot \tilde{R}=K_{\tilde{S}} \cdot \tilde{R}=K_{\tilde{S}}^{2}-2 h^{0}\left(2 K_{\hat{\Sigma}}+\hat{\Delta}\right)=8-2 h^{0}\left(2 K_{\hat{\Sigma}}+\hat{\Delta}\right),
$$

and hence, since $\tilde{F} \cdot \tilde{R}=F \cdot R=8$, we get $h^{0}\left(2 K_{\hat{\Sigma}}+\hat{\Delta}\right)=0$, as desired.

Now we proceed by considering the case $\tilde{F}^{2}>0$. We begin with the following lemma which rules out one of the possibilities in Lemma 2.1.

Lemma 2.3. Suppose $\tilde{F}^{2}>1$. Then $q=0$ and $K_{\tilde{S}}^{2}=1$.

Proof. It is enough to show that Case (3) of Lemma 2.1 cannot occur. To get a contradiction, suppose that $\tilde{F}^{2}=2$ and $\tilde{F} \sim K_{\tilde{S}}$. By Proposition 1.2, we have

$$
\tilde{R} \cdot K_{\tilde{S}}+4=\tilde{\nu}=K_{\tilde{S}}^{2}+4-2 h^{0}\left(2 K_{\hat{\Sigma}}+\hat{\Delta}\right)=6-2 h^{0}\left(2 K_{\hat{\Sigma}}+\hat{\Delta}\right),
$$

which yields $\tilde{R} \cdot \tilde{F}=\tilde{R} \cdot K_{\tilde{S}} \leq 2$.

Let $x_{1}, x_{2}$ be the base points of $\varphi_{*}|F| \subseteq|\tilde{F}|$. Then $\varphi=\phi_{1} \circ \phi_{2}$, where $\phi_{1}: S_{1} \rightarrow \tilde{S}$ is the blow up of $x_{1}$ and $\phi_{2}: S \rightarrow S_{1}$ is the blow up of $x_{2}$. Let $\mathfrak{E}_{i}$ be the exceptional curve of $\phi_{i}, i=1,2$, and denote by $\overline{\mathfrak{E}}_{1}$ the strict transform of $\mathfrak{E}_{1}$ on $S$.

Let $\bar{R}$ be the strict transform of $\tilde{R}$ on $S$. Since $R$ is reduced and $\varphi_{*} R=\tilde{R}$, we have $R=\bar{R}+a_{1} \overline{\mathfrak{E}}_{1}+a_{2} \mathfrak{E}_{2}$, where $a_{i} \in\{0,1\}$, and $\bar{R} \cdot F \leq \tilde{R} \cdot \tilde{F} \leq 2$. Hence

$$
8=R \cdot F=\bar{R} \cdot F+a_{1} \overline{\mathfrak{E}}_{1} \cdot F+a_{2} \mathfrak{E}_{2} \cdot F \leq 2+a_{1} \overline{\mathfrak{E}}_{1} \cdot F+a_{2} \mathfrak{E}_{2} \cdot F,
$$

and thus $\overline{\mathfrak{E}}_{1} \cdot F \geq 2$ or $\mathfrak{E}_{2} \cdot F \geq 2$. But this means that $\tilde{F}$ is singular at $x_{1}$ or $x_{2}$, a contradiction. 
Proposition 2.4. Suppose $\tilde{F}^{2}>0$. Then the bicanonical map of $\tilde{S}$ factors through $\tilde{\sigma}$.

Proof. In order to prove the claim it is sufficient to show that $h^{0}\left(2 K_{\hat{\Sigma}}+\hat{\Delta}\right)=0$.

Suppose $K_{\tilde{S}}^{2}=1$. Then $p_{g}=0$ and, since $K_{\tilde{S}}$ is nef, Proposition 1.2 yields $4 \leq$ $\tilde{R} \cdot K_{\tilde{S}}+4=5-2 h^{0}\left(2 K_{\hat{\Sigma}}+\hat{\Delta}\right)$. Thus $h^{0}\left(2 K_{\hat{\Sigma}}+\hat{\Delta}\right)=0$.

Now assume $K_{\tilde{S}}^{2}>1$. By Lemma 2.3, we have $\tilde{F}^{2}=1$ and so, by the adjunction formula, $K_{\tilde{S}} \cdot \tilde{F}=3$. To get a contradiction, suppose $h^{0}\left(2 K_{\hat{\Sigma}}+\hat{\Delta}\right)>0$. Let

$$
\hat{D} \in\left|\rho^{*}\left(2 K_{\hat{\Sigma}}+\hat{\Delta}\right)\right|=\left|2 \rho^{*}\left(K_{\hat{\Sigma}}+\hat{\Delta}\right)-\rho^{*} \hat{\Delta}\right|=\left|2 K_{\hat{S}}-\hat{R}\right| .
$$

The divisor $D=\pi_{*} \hat{D}$ is effective and linearly equivalent to $2 K_{\tilde{S}}-\tilde{R}=\tilde{\pi}_{*}\left(2 K_{\hat{S}}-\hat{R}\right)$ and so, since $\varphi_{*}|F| \subseteq|\tilde{F}|$ has no fixed component, $D \cdot \tilde{F} \geq 0$. Recall that $\tilde{R}=\varphi_{*} R$.

Since $\tilde{F}^{2}=1, \varphi: S \rightarrow \tilde{S}$ is the blow up of the unique base point of $\varphi_{*}|F|$. Let $E$ be the exceptional curve of $\varphi$. Then

$$
\tilde{R} \cdot \tilde{F}=\varphi_{*} R \cdot \tilde{F}=R \cdot \varphi^{*} \tilde{F}=R \cdot(F+E)=8+R \cdot E .
$$

Since $R$ is reduced, we have $R \cdot E \geq-1$ and so $\tilde{R} \cdot \tilde{F} \geq 7$. But then we get

$$
D \cdot \tilde{F}=2 K_{\tilde{S}} \cdot \tilde{F}-\tilde{R} \cdot \tilde{F}=6-\tilde{R} \cdot \tilde{F}<0,
$$

a contradiction. Therefore $h^{0}\left(2 K_{\hat{\Sigma}}+\hat{\Delta}\right)=0$.

2.2 The case $\tilde{\boldsymbol{F}}^{\mathbf{2}}=\mathbf{0}$. For the rest of the present section we assume $\tilde{F}^{2}=0$. Notice that, since $f: S \rightarrow \mathbb{P}^{1}$ is relatively minimal, $\varphi: S \rightarrow \tilde{S}$ is an isomorphism. Then we are in the situation described in 1.6. Let $\psi: \hat{\Sigma} \rightarrow \Sigma$ be a birational morphism as in 1.6, so that $\hat{\Gamma}=\hat{\rho}(\hat{F}) \cong \mathbb{P}^{1}$ and the following holds.

(i) $\Gamma=\psi_{*} \hat{\Gamma}$ is a ruling of $\Sigma$ and $\Gamma \cdot B=8$, where $B=\psi_{*} \hat{B}$.

(ii) $a_{k}=0$ if $k \geq 6$, i.e., $B$ has at most [5,5]-points and $\bar{a}=\left(a_{2}, a_{3}, a_{4}, a_{5}\right)$.

(iii) If $B$ has a [5,5]-point at $q_{i}$ then $\psi_{1} \circ \cdots \circ \psi_{i}: \Sigma_{i} \rightarrow \Sigma$ is an isomorphism in a neighborhood of $q_{i}$, and the ruling of $\Sigma$ through the image of $q_{i}$ is a component of $B$.

Lemma 2.5. Under the current assumptions, we have $2 a_{5}+a_{3}=\tilde{\nu} \geq 4$.

Proof. Equality $2 a_{5}+a_{3}=\tilde{\nu}$ follows from Lemma 1.4. As for the inequality, by (1.3) we have $\tilde{\nu}=\tilde{R} \cdot K_{\tilde{S}}+4$, and thus $\tilde{\nu} \geq 4$, because $K_{\tilde{S}}$ is nef.

Lemma 2.6. The fibration $f: S \rightarrow \mathbb{P}^{1}$ has at least $a_{5}$ double fibers.

Proof. Suppose $a_{5} \geq 1$. Then $B$ has a [5,5]-point at, say, $q_{i}$. Let $\Gamma_{i} \subset \Sigma_{i}$ be the pull back of the fiber through the image of $q_{i}$. Then $\Gamma_{i}$ is a component of $B_{i}$.

Let $\tilde{\Gamma}_{i}$ be the strict transform of $\Gamma_{i}$ on $\Sigma_{i+1}$. Then $\tilde{\Gamma}_{i} \cap \mathcal{E}_{i+1}=\left\{q_{i+1}\right\}$ and $\tilde{\Gamma}_{i}+\mathcal{E}_{i+1}$ is a component of $B_{i+1}$. Let $\hat{\Gamma}_{i}$ be the pull back of $\Gamma_{i}$ to $\hat{\Sigma}$, and let $\bar{\Gamma}_{i}, \overline{\mathcal{E}}_{i+1}$ be the strict transforms of $\Gamma_{i}$ and $\mathcal{E}_{i+1}$ on $\hat{\Sigma}$, respectively. Then $\hat{\Gamma}_{i} \in|\hat{\Gamma}|$ and we have $\hat{\Gamma}_{i}=$ $\bar{\Gamma}_{i}+\overline{\mathcal{E}}_{i+1}+2 \mathcal{E}_{i+2}^{*}$, where $\bar{\Gamma}_{i}+\overline{\mathcal{E}}_{i+1}$ is a component of $\hat{B}$. Let $\hat{F}_{i}=\hat{\rho}^{*} \hat{\Gamma}_{i}$ be the pull back of $\hat{\Gamma}_{i}$ to $\hat{S}$. Then $\hat{F}_{i}=2\left(\hat{\rho}^{-1}\left(\bar{\Gamma}_{i}\right)+\hat{\rho}^{-1}\left(\overline{\mathcal{E}}_{1+1}\right)+\hat{\rho}^{*} \mathcal{E}_{i+2}^{*}\right) \in|\hat{F}|$, and the curve $\pi_{*} \hat{F}_{i}$ is a double fiber of $f: S \rightarrow \mathbb{P}^{1}$. 
Since $\Sigma$ is rational, $\Sigma \cong \mathbb{P}\left(\mathcal{O}_{\mathbb{P}^{1}} \oplus \mathcal{O}_{\mathbb{P}^{1}}(-e)\right)$ for some $e \geq 0$. Let $C_{0} \subset \Sigma$ be a section with minimal self-intersection. Then $C_{0}^{2}=-e$ and there are $a, b \in \mathbb{N}$ such that $B \in\left|a C_{0}+b \Gamma\right|$. Since $B \cdot \Gamma=8$, we have $a=8$, and, since $B \in|2 \Delta|, b=2 \lambda$ for some $\lambda \in \mathbb{N}$. Recall that $K_{\Sigma} \in\left|-2 C_{0}-(2+e) \Gamma\right|$.

Lemma 2.7. We have

$$
\begin{aligned}
3 K_{\hat{S}}^{2}+24 & =2 a_{3}+2 a_{4}+2 a_{5}, \\
\lambda & =2 e+a_{5}+\frac{1}{2} K_{\hat{S}}^{2}+5 .
\end{aligned}
$$

Proof. Since $a_{k}=0$ if $k \geq 6$, from (1.4) and (1.5) it follows that

$$
\begin{aligned}
1=\chi(\hat{S}) & =\frac{1}{2}\left(2 C_{0}+(\lambda-2-e) \Gamma\right) \cdot\left(4 C_{0}+\lambda \Gamma\right)+2-\frac{1}{2}\left(8 a_{5}+2 a_{4}+2 a_{3}\right) \\
& =-6 e+3 \lambda-2-4 a_{5}-a_{4}-a_{3}, \\
K_{\hat{S}}^{2} & =2\left(2 C_{0}+(\lambda-2-e) \Gamma\right)^{2}-2\left(5 a_{5}+a_{4}+a_{3}\right) \\
& =-16 e+8 \lambda-16-10 a_{5}-2 a_{4}-2 a_{3} .
\end{aligned}
$$

Whence we get $3 K_{\hat{S}}^{2}-8=-32+2 a_{5}+2 a_{4}+2 a_{3}$ and $K_{\hat{S}}^{2}-2=2 \lambda-4 e-12-2 a_{5}$, which lead to (2.4) and (2.5).

Recall that $\hat{f}: \hat{S} \rightarrow \mathbb{P}^{1}$ (respectively $\hat{h}: \hat{\Sigma} \rightarrow \mathbb{P}^{1}$ ) is the fibration $\hat{f}=f \circ \pi$ (respectively $\hat{h}=\hat{f} \circ \hat{\rho}^{-1}$ ) whose general fiber is $\hat{F}$ (respectively $\hat{\Gamma}$ ). The following lemma will be useful.

Lemma 2.8. For every $s \in \mathbb{P}^{1}$, let $\gamma_{\hat{f}}(s)$ be the number of irreducible components of the fiber of $\hat{f}: \hat{S} \rightarrow \mathbb{P}^{1}$ over $s$. Then $\gamma_{\hat{f}}(s)=1$ except for a finite number of points $s \in \mathbb{P}^{1}$, and

$$
\sum_{s \in \mathbb{P}^{1}}\left(\gamma_{\hat{f}}(s)-1\right) \leq 2 p_{g}(\hat{S})+8-K_{\hat{S}}^{2} .
$$

Proof. The general fiber of $\hat{f}$ is smooth by Bertini's theorem. Then $\gamma_{\hat{f}}(s)=1$ except for a finite number of points $s \in \mathbb{P}^{1}$. By [4, Lemme 2] we have

$$
\sum_{s \in \mathbb{B}}\left(\gamma_{\hat{f}}(s)-1\right) \leq \rho(\hat{S})-2,
$$

where $\rho(\hat{S})$ is the dimension of the subspace $N S(\hat{S})_{\mathbb{Q}}$ of $H^{2}(\hat{S}, \mathbb{Q})$ generated by the algebraic classes. Since

$$
\begin{aligned}
\rho(\hat{S}) & \leq b_{2}(\hat{S})-2 p_{g}(\hat{S})=10\left(p_{g}(\hat{S})+1\right)-8 q(\hat{S})-K_{\hat{S}}^{2} \\
& =2\left(p_{g}(\hat{S})+1\right)+8 \chi(\hat{S})-K_{\hat{S}}^{2},
\end{aligned}
$$

where $b_{2}(\hat{S})$ is the second Betti number, we get (2.6). 
Let $\gamma_{\hat{h}}(s)$ be the number of irreducible components of the fiber of $\hat{h}: \hat{\Sigma} \rightarrow \mathbb{P}^{1}$ over $s$. Then $\gamma_{\hat{f}}(s) \geq \gamma_{\hat{h}}(s)$ for every $s \in \mathbb{P}^{1}$, and so, by (2.6), we have

$$
\sum_{s \in \mathbb{P}^{1}}\left(\gamma_{\hat{h}}(s)-1\right) \leq \sum_{s \in \mathbb{P}^{1}}\left(\gamma_{\hat{f}}(s)-1\right) \leq 2 p_{g}(\hat{S})+8 \chi(\hat{S})-K_{\hat{S}}^{2} .
$$

On the other hand, we have

$$
\sum_{s \in \mathbb{P}^{1}}\left(\gamma_{\hat{h}}(s)-1\right)=K_{\Sigma}^{2}-K_{\hat{\Sigma}}^{2}=d
$$

Therefore, by (1.6), we get

$$
a_{2}+2 a_{3}+a_{4}+2 a_{5}=d \leq 2 p_{g}(\hat{S})+8-K_{\hat{S}}^{2}
$$

The following proposition is the key result to handle the case under consideration. In fact, the proof of Theorem 0.1 in the case when $p_{g}=0$ will follow at once from it, and few possibilities will be left to be considered in the case when $p_{g}=1$.

Proposition 2.9. We have

$$
\begin{aligned}
& h^{0}\left(2 K_{\hat{\Sigma}}+\hat{\Delta}\right) \leq \frac{2}{5}\left(2+p_{g}\right), \\
& a_{4} \geq 4 K_{\hat{S}}^{2}+16-2 p_{g} .
\end{aligned}
$$

Proof. We have $\tilde{\nu}=a_{3}+2 a_{5}, d=a_{2}+2 a_{3}+a_{4}+2 a_{5}$, and, by (2.4), $3 K_{\hat{S}}^{2}+24=$ $2 a_{3}+2 a_{4}+2 a_{5}$. Then $3 K_{\hat{S}}^{2}+24 \leq 2 d-\tilde{\nu}$, and so, by (2.7), we obtain

$$
3 K_{\hat{S}}^{2}+24 \leq 4 p_{g}+16-2 K_{\hat{S}}^{2}-\tilde{\nu}
$$

that is

$$
\tilde{\nu} \leq-5 K_{\hat{S}}^{2}+4\left(p_{g}-2\right)
$$

By Proposition 1.2, we have $K_{\hat{S}}^{2}=K_{S}^{2}-\tilde{\nu}=-4+2 h^{0}\left(2 K_{\hat{\Sigma}}+\hat{\Delta}\right)$. Hence

$$
\tilde{\nu} \leq 12+4 p_{g}-10 h^{0}\left(2 K_{\hat{\Sigma}}+\hat{\Delta}\right)
$$

and thus, since $\tilde{\nu} \geq 4$ by Lemma 2.5 ,

$$
h^{0}\left(2 K_{\hat{\Sigma}}+\hat{\Delta}\right) \leq \frac{2}{5}\left(2+p_{g}\right),
$$

i.e. (2.8). As for (2.9), by (2.4) and (2.7) we have

$$
a_{4} \geq a_{4}-a_{2}=3 K_{\hat{S}}+24-d \geq 4 K_{\hat{S}}^{2}+16-2 p_{g} .
$$

This completes the proof. 
The following is an immediate consequence of the above proposition and Proposition 1.2.

Proposition 2.10. If $p_{g}=0$ and $\tilde{F}^{2}=0$, then the bicanonical map of $\tilde{S}$ factors through $\tilde{\sigma}$.

Therefore we are left with the case $p_{g}=q=1$. We begin with the following lemma, which determines the possible values for $\bar{a}=\left(a_{2}, a_{3}, a_{4}, a_{5}\right)$ and $K_{\tilde{S}}^{2}$ when the bicanonical map of $\tilde{S}$ does not factor through $\tilde{\sigma}$.

Lemma 2.11. Assume $q=1$ and $\tilde{F}^{2}=0$. Suppose that the bicanonical map of $\tilde{S}$ does not factor through $\tilde{\sigma}$. Then $h^{0}\left(2 K_{\hat{\Sigma}}^{2}+\hat{\Delta}\right)=1$ and one of the following occurs.

(1) $K_{\tilde{S}}^{2}=4, \bar{a}=(0,0,6,3)$.

(2) $K_{\tilde{S}}^{2}=3, \bar{a}=(0,1,6,2)$.

(3) $K_{\tilde{S}}^{2}=2, \bar{a}=(0,2,6,1)$ or $\bar{a}=\left(a_{2}, 0,7,2\right)$ with $a_{2} \leq 1$.

Proof. By Propositions 1.2 and 2.9, we have $h^{0}\left(2 K_{\hat{\Sigma}}^{2}+\hat{\Delta}\right)=1$. Thus, by (1.2), we get $K_{\hat{S}}^{2}=K_{\tilde{S}}^{2}-\tilde{\nu}=-2$ and from (2.9) it follows that $a_{4} \geq 6$.

Suppose $a_{4} \geq 7$. Then, by (2.4), $a_{5}+a_{3}=9-a_{4} \leq 2$ and we get

$$
4 \leq \tilde{\nu}=2 a_{5}+a_{3} \leq a_{5}+2 .
$$

Whence $a_{5}=2, a_{3}=0, a_{4}=7$ and $\tilde{\nu}=4$. Therefore $K_{\tilde{S}}^{2}=2$ and, by $(2.7), a_{2} \leq 1$.

Suppose $a_{4}=6$. Then, by (2.4), $a_{5}+a_{3}=3$ and as before we get $1 \leq a_{5}$. Note that, by (2.7), we have $a_{2}=0$. Hence, either

- $a_{5}=3, a_{3}=0$ and $K_{S}^{2}=4$, or

- $a_{5}=2, a_{3}=1$ and $K_{S}^{2}=3$, or

- $a_{5}=1, a_{3}=2$ and $K_{S}^{2}=2$.

This completes the proof.

The next step will be to show that possibilities (1) and (2) of Lemma 2.11 cannot occur. For this purpose we need to study the behavior of the curve $\tilde{R} \subset \tilde{S}$.

Recall that

$$
\tilde{\pi}^{*} \tilde{R}=\hat{R}-\sum_{i=1}^{\tilde{\nu}} E_{i},
$$

and $\hat{R} \equiv \hat{\rho}^{*} \hat{\Delta}$. Since $\hat{R}$ is smooth and the $E_{i}$ 's are $(-1)$-curves, $\tilde{R}^{2}=\hat{R}^{2}+\tilde{\nu}=2 \hat{\Delta}^{2}+\tilde{\nu}$. On the other hand

$$
\hat{\Delta} \equiv \psi^{*}\left(4 C_{0}+\lambda \Gamma\right)-\sum\left\lfloor\frac{m_{i}}{2}\right\rfloor \mathcal{E}_{i}^{*},
$$

and so, since $B$ has at most $[5,5]$-points, we get

$$
\hat{\Delta}^{2}=-16 e+8 \lambda-\sum\left\lfloor\frac{m_{i}}{2}\right\rfloor^{2}=-16 e+8 \lambda-\left(13 a_{5}+4 a_{4}+5 a_{3}+a_{2}\right) .
$$

Thus, by (2.5) and (2.4), we have

$$
\hat{\Delta}^{2}=-\left(5 a_{5}+4 a_{4}+5 a_{3}+a_{2}\right)+32=-4-a_{5}-a_{3}-a_{2},
$$


which, by Lemma 2.5, yields

$$
\tilde{R}^{2}=\hat{R}^{2}+\tilde{\nu}=\hat{R}^{2}+2 a_{5}+a_{3}=2 \hat{\Delta}^{2}+2 a_{5}+a_{3}=-8-a_{3}-2 a_{2} .
$$

Write $\tilde{R}=\sum \tilde{R}_{i}+\sum Z_{i}$, where the $\tilde{R}_{i}$ 's are irreducible curves of genus $g_{i}=$ $g\left(\tilde{R}_{i}\right) \geq 1$ and the $Z_{i}$ 's are rational curves. Then, since $\tilde{R}$ is smooth,

$$
K_{\tilde{S}} \cdot \tilde{R}+\tilde{R}^{2}=\sum\left(K_{\tilde{S}} \cdot \tilde{R}_{i}+\tilde{R}_{i}^{2}\right)+\sum\left(K_{\tilde{S}} \cdot Z_{i}+Z_{i}^{2}\right)=\sum\left(2 g_{i}-2\right)-2 \mu,
$$

where $\mu$ denotes the number of rational curves belonging to $\tilde{R}$. Hence we get

$$
2 \mu=\sum\left(2 g_{i}-2\right)-\tilde{R}^{2}-\tilde{R} \cdot K_{\tilde{S}}=\sum\left(2 g_{i}-2\right)+8+a_{3}+2 a_{2}-\tilde{R} \cdot K_{\tilde{S}},
$$

and thus, by (1.3) and Lemma 2.5,

$$
\mu=\sum_{g_{i} \geq 1}\left(g_{i}-1\right)+6-a_{5}+a_{2} .
$$

We recall that, since $\tilde{S}$ is minimal of general type, $Z_{i}^{2} \leq-2$ for any $i$.

Now we are ready to show that Cases (1) and (2) of Lemma 2.11 cannot occur.

Proposition 2.12. Suppose that $q=1$ and $K_{\tilde{S}}^{2}>2$. If $\tilde{F}^{2}=0$, then the bicanonical map of $\tilde{S}$ factors through $\tilde{\sigma}$.

Proof. Suppose to the contrary that the bicanonical map does not factor through $\tilde{\sigma}$. Then $h^{0}\left(2 K_{\hat{\Sigma}}+\hat{\Delta}\right)=1$ and either Case (1) or Case (2) of Lemma 2.11 occurs.

Case (1). Suppose $K_{\tilde{S}}^{2}=4$. Let $\hat{D} \in\left|\hat{\rho}^{*}\left(2 K_{\hat{\Sigma}}+\hat{\Delta}\right)\right|$ and $D=\tilde{\pi}_{*} \hat{D} \in\left|2 K_{\tilde{S}}-\tilde{R}\right|$. By (2.10) and Proposition 1.2, we have

$$
D^{2}=16-4 \tilde{R} \cdot K_{\tilde{S}}+\tilde{R}^{2}=0 .
$$

Hence, since $D \cdot \tilde{F}=\left(2 K_{\tilde{S}}-\tilde{R}\right) \cdot \tilde{F}=0, D \sim a \tilde{F}$ for some non-negative $a \in \mathbb{Q}$.

By Lemma 2.11 we have $a_{2}=a_{3}=0, a_{4}=6$ and $a_{5}=3$. Thus, by (2.11), $\mu \geq 3$. Let $Z_{1}, Z_{2}, Z_{3}$ be distinct rational components of $\tilde{R}$. Since $\tilde{R}$ is smooth, we have

$$
a \tilde{F} \cdot Z_{i}=D \cdot Z_{i}=2 K_{\tilde{S}} \cdot Z_{i}-Z_{i}^{2} \geq 2,
$$

and so $\tilde{F} \cdot Z_{i}>0$ for each $i=1,2,3$.

Now recall that, by Lemma 2.6, $f: \tilde{S}=S \rightarrow \mathbb{P}^{1}$ has at least $a_{5}=3$ double fibers. Hence $\tilde{F} \cdot Z_{i} \equiv 0(\bmod 2)$ and thus, by Hurwitz formula for the covering $Z_{i} \rightarrow \mathbb{P}^{1}$ induced by $f$, we have $\tilde{F} \cdot Z_{i} \geq 4$. But then we get

$$
8=\tilde{F} \cdot \tilde{R} \geq \sum_{i=1}^{3} \tilde{F} \cdot Z_{i} \geq 12,
$$

a contradiction. Therefore this case cannot occur. 
Case (2). Suppose $K_{\tilde{S}}^{2}=3$. By Proposition $1.2, \tilde{R} \cdot K_{\tilde{S}}=1$. Hence, since $K_{\tilde{S}}$ is nef, we can write $\tilde{R}=Z+M$, where $M$ is a smooth irreducible curve with $M \cdot K_{\tilde{S}}=1$, and $Z_{i} \cdot K_{\tilde{S}}=0$ for each component $Z_{i}$ of $Z$. Notice that the $Z_{i}$ 's are $(-2)$-curves. By the algebraic index theorem we have $3 M^{2}=M^{2} K_{\tilde{S}}^{2} \leq 1$. Hence, since by adjunction $M^{2}=2 g(M)-2-M \cdot K_{\tilde{S}} \geq-3$, we get $M^{2} \in\{-1,-3\}$.

By Lemma 2.11 we have $a_{2}=0, a_{3}=1, a_{4}=6$ and $a_{5}=2$. Thus, by (2.10), $\tilde{R}^{2}=-9$ and one of the following occurs.

(†) $Z=Z_{1}+Z_{2}+Z_{3}$ and $M$ is a (-3)-curve,

(‡) $Z=Z_{1}+Z_{2}+Z_{3}+Z_{4}$ and $M$ is a smooth elliptic curve with $M^{2}=-1$.

In any case we have $\tilde{F} \cdot Z_{i}>0$ for each $i$. For if $\tilde{F} \cdot Z_{i}=0$, then, since $\tilde{R}$ is smooth, we get

$$
K_{\tilde{S}}^{2}\left(\tilde{F}+\tilde{R}-Z_{i}\right)^{2}=3\left(16+\tilde{R}^{2}-Z_{i}^{2}\right)=27
$$

and

$$
\left(\tilde{F}+\tilde{R}-Z_{i}\right) \cdot K_{\tilde{S}}=(\tilde{F}+\tilde{R}) \cdot K_{\tilde{S}}=5,
$$

which contradicts the algebraic index theorem.

Note that since $f: \tilde{S}=S \rightarrow \mathbb{P}^{1}$ has at least $a_{5}=2$ double fibers, $\tilde{F} \cdot C \equiv 0(\bmod 2)$ for any curve $C$ on $\tilde{S}$. In particular, since $\tilde{F} \cdot Z_{i}>0$, we have $\tilde{F} \cdot Z_{i} \geq 2$ for every $i$.

Suppose we are in case $(\dagger)$. Then $\tilde{F} \cdot M=\tilde{F} \cdot \tilde{R}-\sum \tilde{F} \cdot Z_{i} \leq 2$, and so $K_{\tilde{S}}^{2}(\tilde{F}+$ $\tilde{R}-M)^{2}=3(10-2 \tilde{F} \cdot M) \geq 18$. On the other hand we have $(\tilde{F}+\tilde{R}-M) \cdot K_{\tilde{S}}=4$, contradicting the algebraic index theorem.

Suppose we are in case $(\ddagger)$. Since $\tilde{R} \cdot \tilde{F}=8$, we have $M \cdot \tilde{F}=0$ and then $\hat{\rho} \circ \tilde{\pi}^{-1}(M)$ is contained in a fiber of $\hat{\Sigma} \rightarrow \mathbb{P}^{1}$. But this is a contradiction. In fact, since $M \subset \tilde{R}$ and $\hat{\rho}$ is finite, $\hat{\rho} \circ \tilde{\pi}^{-1}(M)$ is a curve isomorphic to $M$, and hence elliptic.

Therefore Case (2) of Lemma 2.11 cannot occur. This completes the proof.

\section{Conclusion}

In this section we finish the proof of Theorem 0.1 and establish some consequences of it, such as Theorem 0.2.

Proof of Theorem 0.1 . Let $\varphi_{2 K_{S}}$ (respectively $\varphi_{2 K_{\tilde{S}}}$ ) be the bicanonical map of $S$ (respectively $\tilde{S}$ ).

Since $\varphi: S \rightarrow \tilde{S}$ is a birational morphism, $\varphi_{2 K_{S}}=\varphi_{2 K_{\tilde{S}}} \circ \varphi$ and so, by the definition of $\tilde{\sigma}$, we have that $\varphi_{2 K_{S}}$ factors through $\sigma$ if and only if $\varphi_{2 K_{\tilde{S}}}$ factors through $\tilde{\sigma}$. Therefore the first part of Theorem 0.1 follows from Propositions 2.2, 2.4 and 2.12.

As for the last claim, suppose first that $p_{g} \neq 1$ or $K_{\tilde{S}}^{2} \neq 2$. Then $\varphi_{2 K_{S}}$ factors through the quotient map $S \rightarrow S / \sigma$ and, since $F / \sigma_{F} \cong \mathbb{P}^{1}, S / \sigma$ is birational to a smooth rational ruled surface $W$. Hence $\varphi_{2 K_{S}}$ factors through the $2: 1$ induced map $S \rightarrow W$.

Suppose now that $q=1$ and $K_{\tilde{S}}^{2}=2$. Then, by [13, Theorem 5.1], the Albanese morphism of $\tilde{S}$ is a fibration $\alpha: \tilde{S} \rightarrow \operatorname{Alb}(\tilde{S})$ of genus two over an elliptic curve. Let $\tau \in \operatorname{Aut}(\tilde{S})$ induced by the hyperelliptic involution of the general fibers of $\alpha$. Then 
$\varphi_{2 K_{S}}$ factors through the double cover $S \rightarrow S / \tau$. Since $S / \tau$ is birational to a smooth ruled surface $W$ with $q(W)=1$, we are done.

Proof of Theorem 0.2. Suppose that the bicanonical map of $\tilde{S}$ factors through a generically finite map of degree two onto a ruled surface. By [7, Theorem 0.4$]$, either $\tilde{S}$ presents the standard case, or there exist a birational morphism $\varphi: S \rightarrow \tilde{S}$ and a hyperelliptic fibration $f: S \rightarrow \mathbb{P}^{1}$ of genus 3 . If $\tilde{S}$ presents the standard case, there is a fibration $S \rightarrow \mathbb{B}$ of genus 2 and a birational morphism $\varphi: S \rightarrow \tilde{S}$. Since $\mathbb{B}$ has genus $b \leq q(S)=q(\tilde{S})$, we are done.

As for the converse, let $g$ be the genus of a general fiber of $f: S \rightarrow \mathbb{B}$. If $g=3$, the claim follows from Theorem 0.1. Whereas if $g=2$, it is well known that the bicanonical map factors through a double cover onto a ruled surface.

The following corollary is a characterization of minimal surfaces with $p_{g}=0, K^{2} \geq 6$ and nonbirational bicanonical map.

Corollary 3.1. Let $\tilde{S}$ be a minimal surface of general type with $p_{g}(\tilde{S})=0$. Assume that $K_{\tilde{S}}^{2} \geq 6$. Then the following are equivalent.

(1) The bicanonical map of $\tilde{S}$ is nonbirational.

(2) There exists a fibration $f: \tilde{S} \rightarrow \mathbb{P}^{1}$ whose general fiber is a smooth hyperelliptic curve of genus 3.

Proof. The implication (2) $\Rightarrow(1)$ follows from Theorem 0.1. Implication $(1) \Rightarrow(2)$ is proved by M. Mendes Lopes and R. Pardini in the following papers, [14], [15], [16], [19] and [17].

\section{The examples}

There exist several examples in the literature of surfaces of general type with $p_{g}=q$. All the examples below have already appeared in literature. We will only describe briefly the construction, explaining how one recovers the hyperelliptic fibration $f: S \rightarrow \mathbb{P}^{1}$ of genus 3 . For the details we refer to the original papers where they have been presented.

Surfaces with $p_{g}=q=\mathbf{0}$.

4.1 Burniat's examples. ([8], cf. also [20]) These are minimal smooth models of bidouble covers of $\mathbb{P}^{2}$, with $K_{\tilde{S}}^{2}=6-m, 0 \leq m \leq 4$. It can be easily checked that these surfaces have an hyperelliptic fibration of genus 3 .

4.2 Numerical Godeaux surfaces. There are several example of numerical Godeaux surfaces, i.e. minimal surfaces of general type with $p_{g}=q=0$ and $K_{\tilde{S}}^{2}=1$. An example of such a surface admitting a hyperelliptic fibration of genus 3 over $\mathbb{P}^{1}$ has been constructed in [9]. This is the smooth minimal model of the double cover of $\mathbb{P}^{2}$ branched along a reduced curve $B$ of degree 14 with certain singularities. Without entering in deep 
details about the singularities of $B$, we just mention that $B$ has multiplicity 6 at a point $p \in \mathbb{P}^{2}$. Then the pencil of lines through $p$ pulls back to $\tilde{S}$ to a pencil of hyperelliptic curves of genus 3 .

4.3 Mendes Lopes and Pardini's examples. M. Mendes Lopes and R. Pardini studied in [14] minimal surfaces of general type with $p_{g}(\tilde{S})=0, K_{\tilde{S}}^{2}=7,8$, and nonbirational bicanonical map. Among other things, they constructed examples of these surfaces, showing that they admit a fibration over $\mathbb{P}^{1}$ with general fiber a hyperelliptic curve of genus 3 .

Surfaces with $p_{g}=q=1$.

4.4 Stagnaro's example. E. Stagnaro constructs in [27, Example 2] a minimal surface $S$ of general type with $p_{g}=q=1$ and $K_{\tilde{S}}^{2}=3 . \quad \tilde{S}$ is the smooth minimal model of the double cover of $\mathbb{P}^{2}$ branched along a reduced curve $B$ of degree 12 with certain singularities. We just mention that $B$ has a [5,5]-point at a point $p \in \mathbb{P}^{2}$. Then the pencil of lines through $p$ pulls back to a pencil of hyperelliptic curves of genus 3 . In this case, denoting by $F$ a general member of the pencil, one has $F^{2}=1$.

4.5 Polizzi's examples. Let $G=D_{4}$ be the dihedral group of order 8, i.e., the group of symmetries of a square. Let $r$ be the rotation. In [23] and [24] F. Polizzi shows that for any $g \in\{2,3,4,5\}$ there exist a curve $F$ of genus 3 and a curve $C$ of genus $g$ such that

- $G$ acts on $F$ and $C$, and hence acts diagonally on the product $F \times C$, i.e. $\mathfrak{g}(x, y)=$ $(\mathfrak{g}(x), \mathfrak{g}(y))$, for $\mathfrak{g} \in G$ and $(x, y) \in F \times C$,

- $F / G$ (respectively $C / G$ ) is a smooth elliptic (respectively rational) curve,

- the minimal desingularization of the quotient $(F \times C) / G$ is a minimal surface of general type $\tilde{S}$ with $p_{g}(\tilde{S})=q(\tilde{S})=1$ and $K_{\tilde{S}}^{2}=2 g-2 \in\{2,4,6,8\}$.

Let $f: \tilde{S} \rightarrow C / G \cong \mathbb{P}^{1}$ and $h: \tilde{S} \rightarrow F / G$ be the fibrations induced by the two projections $F \times C \rightarrow C$ and $F \times C \rightarrow F$. Then the general fiber of $f$ (respectively $h$ ) is isomorphic to $F$ (respectively $C$ ). Notice that, since $F / G$ is an elliptic curve and $q(\tilde{S})=1, h: \tilde{S} \rightarrow F / G$ is the Albanese morphism of $\tilde{S}$. The subgroup $H \subset G$ generated by $r^{2}$ acts on $F$ without fixed points. It follows that $F / H$ is a smooth curve of genus 2, and hence $F$ is hyperelliptic (cf. [1, Lemma 5.10]).

Polizzi kindly communicated to us that he and E. Mistretta have recently proved the existence of some surfaces with $p_{g}=q=1$ and $K^{2}=5$ ([18]). They show that there exist smooth curves $F, C$ of genus 3 such that the group $G=S_{3}$ acts diagonally on the product $F \times C$, and $F / G$ (respectively $C / G$ ) is a rational (respectively elliptic) curve. Moreover, the permutation $(12) \in G$ acts on $F$ without fixed points. The minimal desigularization of $(F \times C) / G$ is then a minimal surface of general type $\tilde{S}$ with $p_{g}=q=1$ and $K_{\tilde{S}}^{2}=5$. Since the permutation (12) acts freely on $F$, the general fiber of the induced fibration $f_{\tilde{S}}: S \rightarrow C / G \cong \mathbb{P}^{1}$ is a hyperelliptic curve of genus 3 . Moreover the induced fibration $\tilde{S} \rightarrow F / G$, whose general fiber is isomorphic to $C$, is the Albanese morphism of $\tilde{S}$.

4.6 Remark. By Theorem 0.1, the bicanonical map of Polizzi's examples is nonbirational. Note that, since $q=1$, for all of them the Albanese morphism $\alpha: \tilde{S} \rightarrow \operatorname{Alb}(S)$ 
is the only fibration over a curve of positive genus. As we observed, the general fiber of $\alpha$ is isomorphic to $C$. It follows that if $K_{\tilde{S}}^{2} \in\{4,5,6,8\}$, these are examples of surfaces with nonbirational bicanonical map not presenting the standard case. Indeed $C$ has genus at least 3 and, since $p_{g}(\tilde{S})=q(\tilde{S})=1$ and $K_{\tilde{S}}^{2} \geq 4$, there are no fibrations $\tilde{S} \rightarrow \mathbb{P}^{1}$ of genus 2 (cf. [28, Corollaire 3, p. 51]).

Surfaces with $p_{g}=q=2$.

4.7. In [11, Example 3.13 (c), p. 71] Ciliberto observed that a surface with $p_{g}=q=2$ whose Albanese morphism is not surjective can be constructed as follows. Consider two irreducible smooth curves $F$ and $C$ of genus 3 and 2, such that $F$ is the étale double cover of a smooth curve $D$ of genus two. Then $G=\mathbb{Z}_{2}$ acts on both $F$ and $C$, freely on $F$, so that $F / G$ has genus 2 and $C / G$ is rational. Hence $G$ acts diagonally on the product $F \times C$, and the action is free. The quotient $\tilde{S}=(F \times C) / G$ is a smooth surface of general type with $\chi(\tilde{S})=1$. The general fibers of the induced fibrations $f: \tilde{S} \rightarrow C / G \cong \mathbb{P}^{1}$ and $h: \tilde{S} \rightarrow F / G \cong D$ are respectively isomorphic to $F$ and $C$. Since $D$ has genus 2, $q(\tilde{S}) \geq 2$. Moreover, since $F$ is a double cover of $D$, it is a hyperelliptic curve of genus 3 (cf. [1, Lemma 5.10]). Then $p_{g}=q=2$ and $K_{\tilde{S}}^{2}=8$.

\section{References}

[1] R. D. M. Accola, Topics in the theory of Riemann surfaces. Springer 1994. MR1329541 (97k:30053) Zbl 0820.30002

[2] I. C. Bauer, F. Catanese, R. Pignatelli, Complex surfaces of general type: some recent progress. In: Global aspects of complex geometry, 1-58, Springer 2006. MR2264106 (2007i:14039) Zbl 1118.14041

[3] A. Beauville, L'application canonique pour les surfaces de type général. Invent. Math. 55 (1979), 121-140. MR553705 (81m:14025) Zbl 0403.14006

[4] A. Beauville, Le nombre minimum de fibres singulieres d'une courbe stable sur $P^{1}$. volume 86 of Astérisque, 97-108, Soc. Math. France 1981. Zbl 0502.14009

[5] A. Beauville, L'inégalités $p_{g} \geq 2 q-4$ pour les surfaces de type général. Appendix to [12]. Bull. Soc. Math. France 110 (1982), 343-346. MR688038 (84f:14026) Zbl 0543.14026

[6] E. Bombieri, Canonical models of surfaces of general type. Inst. Hautes Études Sci. Publ. Math. no. 42 (1973), 171-219. MR0318163 (47 \#6710) Zbl 0259.14005

[7] G. Borrelli, The classification of surfaces of general type with nonbirational bicanonical map. J. Algebraic Geom. 16 (2007), 625-669. MR2357686 (2008m:14074) Zbl 1132.14036

[8] P. Burniat, Sur les surfaces de genre $P_{12} \geq 1$. Ann. Mat. Pura Appl. (4) 71 (1966), 1-24. MR0206810 (34 \#6626) Zbl 0144.20203

[9] A. Calabri, C. Ciliberto, M. Mendes Lopes, Numerical Godeaux surfaces with an involution. Trans. Amer. Math. Soc. 359 (2007), 1605-1632. MR2272143 (2007j:14059) Zbl 1124.14036

[10] F. Catanese, R. Pignatelli, Fibrations of low genus. I. Ann. Sci. École Norm. Sup. (4) 39 (2006), 1011-1049. MR2316980 (2008g:14014) Zbl 1125.14023 
[11] C. Ciliberto, The bicanonical map for surfaces of general type. In: Algebraic geometrySanta Cruz 1995, volume 62 of Proc. Sympos. Pure Math., 57-84, Amer. Math. Soc. 1997. MR1492518 (98m:14040) Zbl 0929.14022

[12] O. Debarre, Inégalités numériques pour les surfaces de type général. Bull. Soc. Math. France 110 (1982), 319-346. MR688038 (84f:14026) Zbl 0543.14026

[13] E. Horikawa, Algebraic surfaces of general type with small $c_{1}^{2}$. V. J. Fac. Sci. Univ. Tokyo Sect. IA Math. 28 (1981), 745-755 (1982). MR656051 (84d:14019) Zbl 0505.14028

[14] M. Mendes Lopes, R. Pardini, The bicanonical map of surfaces with $p_{g}=0$ and $K^{2} \geq 7$. Bull. London Math. Soc. 33 (2001), 265-274. MR1817764 (2002a:14042) Zbl 1075.14514

[15] M. Mendes Lopes, R. Pardini, A connected component of the moduli space of surfaces with $p_{g}=0$. Topology 40 (2001), 977-991. MR1860538 (2002g:14052) Zbl 1072.14522

[16] M. Mendes Lopes, R. Pardini, The bicanonical map of surfaces with $p_{g}=0$ and $K^{2} \geq 7$. II. Bull. London Math. Soc. 35 (2003), 337-343. MR1960943 (2004a:14040) Zbl

[17] M. Mendes Lopes, R. Pardini, Surfaces of general type with $p_{g}=0, K^{2}=6$ and non birational bicanonical map. Math. Ann. 329 (2004), 535-552. MR2127989 (2005m:14067) Zbl 1061.14034

[18] E. Mistretta, F. Polizzi, Standard isotrivial fibrations with $p_{g}=q=1$. II. Eprint 0805.1424

[19] R. Pardini, The classification of double planes of general type with $K^{2}=8$ and $p_{g}=0 . J$. Algebra 259 (2003), 95-118. MR1953710 (2004a:14041) Zbl 1066.14046

[20] C. A. M. Peters, On certain examples of surfaces with $p_{g}=0$ due to Burniat. Nagoya Math. J. 66 (1977), 109-119. MR0444676 (56 \#3026) Zbl 0342.14018 Zbl 0329.14019

[21] G. P. Pirola, Curves on generic Kummer varieties. Duke Math. J. 59 (1989), 701-708. MR1046744 (91b:14029) Zbl 0717.14021

[22] F. Polizzi, On surfaces of general type with $p_{g}=q=1, K^{2}=3$. Collect. Math. 56 (2005), 181-234. MR2154303 (2006d:14038) Zbl 1084.14038

[23] F. Polizzi, Surfaces of general type with $p_{g}=q=1, K^{2}=8$ and bicanonical map of degree 2. Trans. Amer. Math. Soc. 358 (2006), 759-798. MR2177040 (2006j:14051) Zbl 1083.14043

[24] F. Polizzi, Standard isotrivial fibrations with $p_{g}=q=1$. J. Algebra 321 (2009), 1600-1631.

[25] I. Reider, Vector bundles of rank 2 and linear systems on algebraic surfaces. Ann. of Math. (2) 127 (1988), 309-316. MR932299 (89e:14038) Zbl 0663.14010

[26] C. Rito, On surfaces with $p_{g}=q=1$ and non-ruled bicanonial involution. Ann. Sc. Norm. Super. Pisa Cl. Sci. (5) 6 (2007), 81-102. MR2341516 (2008i:14058) Zbl pre05177567

[27] E. Stagnaro, Numerical Burniat and irregular surfaces. Acta Appl. Math. 75 (2003), 167-181. MR1975566 (2004h:14043) Zbl 1062.14049

[28] G. Xiao, Surfaces fibrées en courbes de genre deux. Springer 1985. MR872271 (88a:14042) Zbl 0579.14028

[29] G. Xiao, $\pi_{1}$ of elliptic and hyperelliptic surfaces. Internat. J. Math. 2 (1991), 599-615. MR1124285 (92j:14049) Zbl 0762.14013

Received 16 October, 2007; revised 21 August, 2008

G. Borelli, Departamento de Matemática, Universidade Federal de Pernambuco, Cidade Universitária, 50670-901 Recife (PE), Brasil

Email: borrelli@dmat.ufpe.br 